\title{
¿Es Todavía Lucrativo Cultivar Lichi en la Florida?1
}

\author{
Edward Evans, Robert Degner, Jonathan Crane, Ray Rafle, and Carlos Balerdi ${ }^{2}$
}

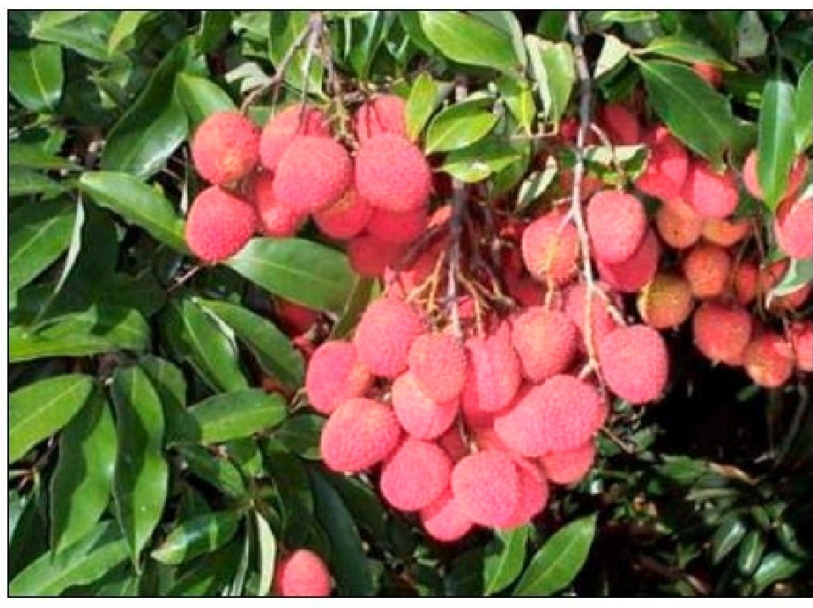

Introducción

Los cinco países más importantes del mundo en la produccción de lichi son China, India,

Taiwán, y Vietnam. El lichi es también cultivado comercialmente en muchas otras áreas subtropicales, incluyendo Israel, Australia, Madagascar, partes de Africa y partes elevadas en Méjico, America Central y Sudamérica. Una cantidad relativamente pequeña es producida en los Estados Unidos (FAO, 2002).
La producción mundial de lichi es estimada en alrededor de 2.11 millones de toneladas, con más del $95 \%$ proveniente de Asia. La producción total en el hemisferio sur (principalmente Africa, Madagascar y Australia) es de más o menos 50,000 toneladas. La época de producción en el hemisferio norte se extiende de Abril hasta mediados de Agosto, y en el hemisferio sur de Noviembre a Febrero.

En los Estados Unidos, las plantaciones comerciales de lichi se encuentran en Florida, Hawaii y California. Florida es el mayor productor de lichi en los Estados Unidos (1,200 acres), seguido por Hawaii (300 acres) y California (60 acres). Se estima que la producción anual de lichi en los Estados Unidos es de 433 toneladas, la que se considera minúscula en comparación a la producción mundial.

La produccción de lichi en Florida se expandió rápidamente después del huracán Andrew en 1992. De 200 acres antes del huracán creció a 500 acres en 1996 y llegó al presente nivel en el 2000 (Degner, Stevens y Morgan, 2002). Los productores fueron atraídos por las altas ganacias y los

1. Este documento, FE547, es uno de una serie de publicaciones del Departamento de Food and Resource Economics, Servicio de Extensión Cooperativa de la Florida, Instituto de Alimentos y Ciencias Agrícolas, Universidad de la Florida (UF/IUFAS). Fecha de primera publicación: Abril 7, 2005. Este traducción del FE496, publicado Noviembre 2004. La traducción del inglés al español estuvo a cargo de Carols Jauregui. Visite nuestro sitio web EDIS en $<$ http://edis.ifas.ufl.edu>.

2. Edward Evans, Assistant Professor, Department of Food and Resource Economics, Tropical Research and Education Center, Homestead, FL; Robert Degner, Professor and Director of the Florida Agriucltural Market Research Center, Department of Food and Resource Economics, University of Florida, Gainesville, FL; Jonathan Crane, Professor, Department of Horticultural Sciences, Tropical Research and Education Center, Homestead, FL; Ray Rafle Miami-Dade County South Extension Office, Homestead, FL; and Carlos Balerdi, Miami-Dade County South Extension Office, Homestead, FL, Florida Extension Service, Institute of Food and Agricultural Sciences, University of Florida, Gainesville, FL.

El Instituto de Alimentos y Ciencias Agrícolas es un empleador que opera bajo Acción Afirmativa y provee Oportunidades Igualitarias, autorizado a proveer investigación, información educativa y otros servicios, únicamente a los individuos e instituciones que operan sin discriminación alguna con relación al credo, color, religión, edad, incapacidad, sexo, orientación sexual, estado civil, nacionalidad, opinion política o afiliaciones. Para más información sobre como obtener otras publicaciones de extensión, comuníquese con la oficina de Servicio de Extensión de su condado. Servicio de Extensión de la Florida / Instituto de Alimentos y Ciencias Agrícolas / Universidad de la Florida / Larry Arrington, Decano. 
prospectos de fuertes demandas provenientes de una población asiática creciente en los Estados Unidos. Sin embarago, con los precios actuales desplomándose debajo de los precios obtenidos hace tres a cinco años y con los costos de producción incrementándose continuamente, muchos productores se preguntan si todavía tiene sentido cultivar lichi. En el próximo análisis nosotros examinamos el desarrollo de la industria del lichi dentro de los Estados Unidos y nos enfocamos en los costos y retornos del lichi en Florida para determinar si los productores pueden todava obtener ganacias cultivando el lichi.

\section{Demanda de Lichi}

La demanda de lichi fresco en los Estados Unidos se ha incrementado considerablemente en años recientes. Esto se debe en parte al crecimiento de la población asiática en los Estados Unidos y a los consumidores conscientes de su salud que compran la fruta en tiendas especializadas (Figura 1).

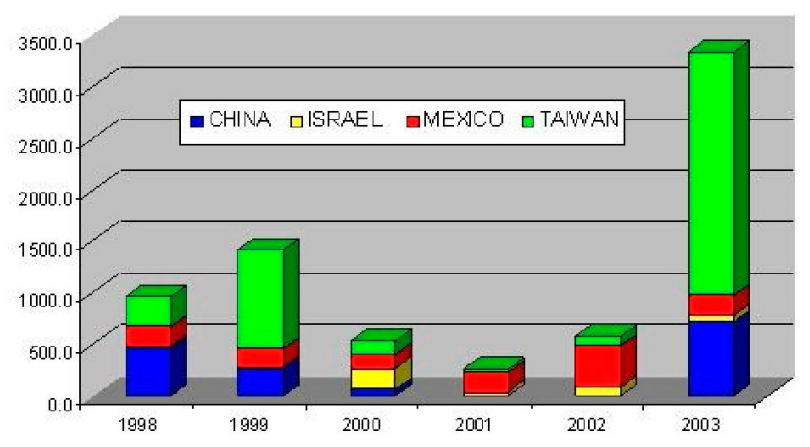

Figure 1. Importaciones americans de lichi, 1998-2003 (toneladas).

En el pasado, la demanda fue principalmente de fruta congelada y enlatada, pero la demanda for fruta fresca se ha incrementado substancialmente. Los principales proveedores de lichi de los Estados Unidos son Taiwán, Méjico, China e Israel. En años recientes, la producción de lichi en los Estados Unidos ha enfrentado una competencia muy fuerte. Entre 1998 y el 2003, la importación de lichi creció de 967.9 a 3,345.8 toneladas, con la mayor parte proveniente de Taiwán. En el 2002, Taiwán dobló sus exportaciones a los Estados Unidos y ahora ha sobrepasado a Méjico como el principal proveedor del mercado maericano. En 2003, Taiwán incrementó sus exportaciones de lichi al nivel más alto, 2,373 toneladas, lo que representa el $70 \%$ de las importaciones americanas de lichi (lo que ha causado que el precio doméstico de lichi en los Estados Unidos se desplome a nuevos niveles).

\section{Precios a la Entrada de los Huertos}

Aparte de un ligero recobro en el 2002, los precios a la entrada de los huertos bajaron en el periodo 1998-2003 (Figura 2). Del nivel alto de $\$ 5.10$ por libra recibido por los productores en 1998 , los precios bajaron hasta aproximadamente $\$ 1.10$ en el 2003, lo que representa una disminución del $78.7 \%$.

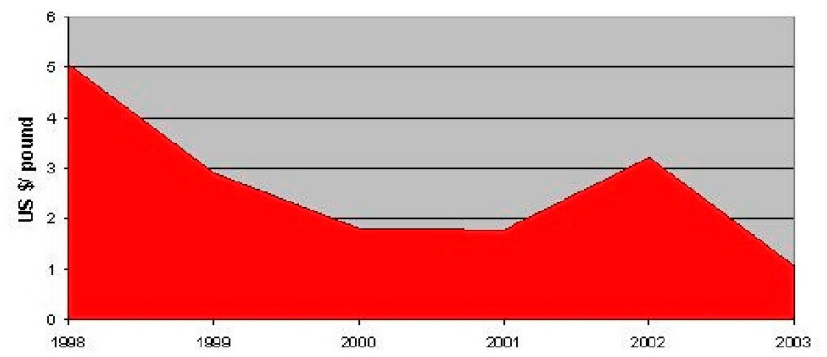

Figure 2. Precios domésticos americanos de lichi a la entrada de los huertos, 1998-2003.

La disminución más grande en los precios se produjo en el 2003 cuando los productores recibieron solo un $33.4 \%$ del precio del año anterior o un $36.4 \%$ del promedio de los precios de cinco años anteriores que fue de \$2.96 por libra. Por primera vez los productores applicaron para obtener asistencia de ajuste comercial, también conocida como TAA (el Acta de Comercio del 2002 estableció la Asistencia de Ajuste Comercial para agricultores, la que permite al Departamento de Agricultura de los Estados Unidos proveer asistencia técnica y dinero en efectivo a agricultores y pescadores que cumplan con requisitos). Como se mencionó anteriormente, la disminución marcada del precio en el mercado ha sido atribuída al incremento en las importaciones de lichi taiwanese y en menor grado al importe de lichi mejicano. Aunque se espera que las importaciones provenientes de Taiwán se estabilizen al nivel actual, los precios a la entrada de los huertos en los Estados Unidos permanecerán débiles en el futuro previsible debido a que hay prospectos de que China entre nuevamente al mercado americano. 


\section{Costos de Producción}

El cuadro 1 ilustra una muestra del presupuesto basado en una producción promedio de 5 años de 5,000 libras de lichi por acre (asumiendo un empacado del $70 \%$ ) y sobre un promedio estimado del precio franco a bordo (F.O.B.) en el sur de Florida. Se debe mencionar que producciones y costos de operaciones individuales pueden variar ampliamente. Por ejemplo, la producción de lichi varía de 600 libras por acre en un año malo hasta 10,000 libras por acre en un buen año. Suposiciones adicionales incluyen cargos de cosecha, transportación y empaque de 50 centavos por libra. Se asume que las empacadoras applican un cargo de ventas de $10 \%$ del precio franco a bordo (F.O.B.).

El cuadro 1 también revela un costo promedio total de producción por acre y comercialización de 6,385 y un ingreso bruto de $\$ 8,750$, dando un retorno neto de $\$ 2,365$. Esto representa un retorno del $37 \%$ sobre las inversiones y los costos recurrentes. Los costos totales de precosecha se estiman en $\$ 3,010$ por acre (alrededor de $\$ 0.60$ por libra) mientras que los costos totales de cosecha y comercialización son de $\$ 3,375$ por acre (aproximadamente $\$ 0.68$ por libra). El cuadro 1 muestra que los mayores costos son de cosecha y comercialización, llegando a ser hasta el $52 \%$ del costo total de producción. La reducción de estos costos contribuira a la mejorara de los retornos.

Adems de reducir los costos de producción para mejorar los ingresos, mejorar la calidad del podría influenciar el precio en el mercado. Los retornos pueden ser mejorados extensivamente si se incrementa la producción por acre (comentado más adelante). El cuadro 2 muestra los retornos netos estimados para varias combinaciones de precios y producciones en el sur de Florida. Las producciones escogidas reflejan las cosechas bajas, medias y altas mientras que los precios reflejan el rango pagado en años recientes. El cuadro 2 revela también que con una cosecha de 3,000 libras o menos, el productor estaría operando a pérdida si el precio franco a bordo (F.O.B.) fuera de $\$ 1.50$ por libra. Sin embargo, si el precio F.O.B. se incrementara de \$1.50 a \$2.00 por libra los retornos netos mejoraran considerablemente aún si la producción se mantuviera a este nivel. En situaciones cuando la producción es alta (7,000 libras por acre) y el precio es cerca de $\$ 2$ por libra, los productores podrían obtener retornos netos de más de $\$ 6,000$. Como la tabla 2 lo mueátra, los retornos de los huertos de lichi pueden variar considerablemente, dependiendo en la producción y el precio recibido. También se ve claramente que un aumento (disminución) del precio en el mercado tiene un mayor impacto de lo que tendría un aumento disminución) similar en la producción. Por ejemplo, aumentando el precio de $\$ 1.50$ a $\$ 2.00$, con una producción de 5,000 libras por acre (aumento del 33.3\%), las ganancias netas aumentarían en $\$ 2,250$, mientras que si la producción aumenta de 5,000 a 7,000 libras por acre (aumento del 40\%) las ganacias netas aumentarían en $\$ 1,700$ (de $\$ 1,240$ a $\$ 2,940$ ). No obstante, realizar semejantes mejoras a través de la reducción en costos sería extremadamente difcil.

\section{Conclusiones}

Nuestro análisis muestra que con una producción de 5,000 libras por acre y un precio F.O.B. de $\$ 1.75$, es posible obtener un retorno neto de $\$ 2,365$ por acre o retornos a la inversion y a los costos recurrentes de un 37\%. Superficialmente, semejantes retornos parecen atractivos. Sin embargo, cuando se considera que el valor presente de un flujo de retornos semejantes sobre 20 años (asumiendo una tasa de interés constante del $6 \%$ al año) es solo de $\$ 27,000$ comparado al valor presente de $\$ 55,000$ por acre de tierra agrícola en el área, se ve la razón por la que muchos agricultores se preguntan si todavía es lucrativo este negocio. Desde otro punto de vista, si un productor obtiene un préstamo hoy día (asumiendo una tasa de interés del 6\% y un período de pago de 20 años) para comprar un acre de tierra para cultivar lichi, tendría que pagar $\$ 4,795$ al año por 20 años. Esta cantidad excede grandemente a los $\$ 2,365$ que obtendría como retorno neto $[\$ 2,815 \mathrm{si}$ el arrendamientoes devuelto $(\$ 2,365+450)=\$ 2,815]$. Aún más, semejantes cálculos no toman en cuenta los costos de establecimiento en los primeros años cuando los árboles no producen. Por lo tanto, semejante inversión sólo puede ser justificada por motivos de especulación en el precio de la tierra. 
Nuestro análisis también muestra que los

retornos netos son más sensitivos a los cambios de precio en el mercado y a los cambios en la producción que a los esfuerzos para reducir costos de producción.

\section{Referencias}

Degner, R.L., T.J. Stevens, III, and K.L. Morgan. 2002. Miami-Dade County Agricultural Land Study. Florida Agricultural Market Research Center, Department of Food and Resource Economics, University of Florida, Gainesville, FL.

http://www.agmarketing.ifas.ufl.edu.

FAO. 2002. Lychee Production in the Asian-Pacific Region. Bangkok, Thailand: RAP Publications. 
Table 1. Costos y retornos estimados de lichi en el condado de Miami-Dade County, FL, 2004

\begin{tabular}{|c|c|c|}
\hline Cataegoría & dólares/Acre/Año & dólares/libra \\
\hline \multicolumn{3}{|l|}{ Producción vendible (5,000 libras por acre) } \\
\hline Precio franco a bordo (F.O.B.) en el sur de Florida & & 1.75 \\
\hline Ingreso total & 8,750 & \\
\hline Costos de operación & 1,041 & \\
\hline Fertilizantes & 429 & \\
\hline Fungicidas & 260 & \\
\hline Herbicidas & 85 & \\
\hline Insecticidas & 117 & \\
\hline Interés sobre el capital de operaciones & 150 & \\
\hline Misceláneos & 689 & \\
\hline Limpieza y preparación del terreno & 8 & \\
\hline Reemplazo de árboles & 14 & \\
\hline Poda de árboles & 219 & \\
\hline Plantación & 4 & \\
\hline Irrigación & 135 & \\
\hline Cortar malezas entre los árboles & 80 & \\
\hline Mano de obra & 229 & \\
\hline Costos fijos & 1,280 & \\
\hline Arrendamiento de la tierra* & 450 & \\
\hline Supervisión & 176 & \\
\hline Gastos generales & 304 & \\
\hline Depreciación y raparo de equipo & 350 & \\
\hline COSTOS TOTALES DE PRECOSECHA & 3,010 & 0.60 \\
\hline Cosecha y costos de comercialización & 3,375 & \\
\hline Cosecha transporte y empaque (\$0.50/libra) & 2,500 & 0.5 \\
\hline Cargo de ventas (10\% del precio F.O.B. de $\$ 1.75$ ) & 875 & 0.18 \\
\hline COSTO TOTAL & 6,385 & 1.28 \\
\hline RETORNO NETO (Ingreso total menos costo total) & 2,365 & 0.47 \\
\hline
\end{tabular}


¿Es Todavía Lucrativo Cultivar Lichi en la Florida?

Table 2. Retornos netos estimados de varias combinaciones de precios y producciones de lichi en el sur de Florida, 2004.

\begin{tabular}{|c|c|c|c|}
\hline & \multicolumn{3}{|c|}{$\begin{array}{c}\text { Precio franco a bordo (F.O.B.), Homestead } \\
\text { (U.S. dólares por libra) }\end{array}$} \\
\hline & $\$ 1.50$ & $\$ 1.75$ & $\$ 2.00$ \\
\hline $\begin{array}{l}\text { Producción } \\
\text { (libras por acre) }\end{array}$ & \multicolumn{3}{|c|}{$\begin{array}{l}\text { Retornos Por Acre } \\
\text { (U.S. dólares) }\end{array}$} \\
\hline 3,000 & -460 & 215 & 890 \\
\hline 5,000 & 1,240 & 2,365 & 3,490 \\
\hline 7,000 & 2,940 & 4,515 & 6,090 \\
\hline
\end{tabular}

